\title{
Proposta de Mecanismo para Replicação de Comportamento de Nuvem para Pesquisa e Reprodutibilidade *
}

\author{
Douglas A. Clementino ${ }^{1}$, Mariana Carmin ${ }^{1}$, Alexander Ditter ${ }^{2}$, Marco A. Z. Alves ${ }^{1}$ \\ ${ }^{1}$ Departamento de Informática \\ Universidade Federal do Paraná (UFPR) - Curitiba - Brasil \\ ${ }^{2}$ Department of Chemical and Biological Engineering \\ Friedrich-Alexander-Universität Erlangen-Nürnberg - Erlangen - Deutschland \\ ${ }^{1}$ \{dac17, mcarmin, mazalves\}@inf.ufpr.br, ${ }^{2}$ alexander.ditter@fau.de
}

\begin{abstract}
The cloud systems, as an important provider of scalable online services, led to manifold research opportunities. This work presents a tool that allows the monitoring, characterization, and reproduction of activities and consumption of CPU, RAM memory, and network traffic in an Infrastructure as a Service cloud environment, presenting means to supports research and reinforces the reproducible aspect in applying the scientific method.
\end{abstract}

Resumo. O sistema de nuvem, como importante fornecedor de serviços escaláveis online, trouxe consigo um novo campo de pesquisa. Este trabalho propõe uma ferramenta que efetue o monitoramento, a caracterização e a reprodução das atividades e consumo de CPU, memória RAM e tráfego de rede de um ambiente de nuvem Infrastructure as a Service, apresentando uma solução que apoia a pesquisa e o caráter reprodutível do método científico.

\section{Introdução}

Os serviços de nuvem, pela definição do National Institute of Standards and Technology (NIST) [Mell and Grance 2011] tem como características a provisão de serviços de forma onipresente, gerenciando um conjunto de recursos de forma a atender requisições sob demanda. Essas características e a busca do mercado pela substituição de produtos por serviços fez com que a arquitetura em nuvem tenha uma posição de destaque tanto comercial quanto acadêmica. Dentro deste cenário, um pesquisador da área de nuvens de Infrastructure as a Service (IaaS), que deseja colocar em prova a sua proposta possui basicamente duas opções: Aplicar sua proposta em um serviço público, que é hospedado por uma entidade terceirizada e possui pluralidades em acesso, requisições e utilização do serviço, mas que necessita de acesso privilegiado para a aplicação de mudanças em sua estrutura de funcionamento (o que também pode acarretar em problemas para o sistema); Ou montar a sua própria nuvem privada com máquinas que estejam a sua disposição, onde tal pesquisador terá total liberdade para aplicar quaisquer mudanças ao seu ambiente, porém que não contém nem a diversidade nem a demanda de serviços existentes em um sistema público, o que pode ser de grande importância para o seu projeto.

Dessa forma, propõe-se uma ferramenta que forneça ao pesquisador um ambiente ideal para o desenvolvimento de sua pesquisa. Por meio do monitoramento do uso de

${ }^{*}$ Este trabalho é parcialmente suportado pela CAPES e Instituto Serrapilheira (grant Serra-1709-16621). 
$C P U$, consumo de memória e tráfego de rede de um sistema de nuvem pública. Pretendese caracterizar essas métricas em traços que descrevem cargas de trabalho, e posteriormente disponibilizar tais traços para o uso do pesquisador. Logo, este pesquisador poderá reproduzir os traços, gerando cargas de trabalho sintéticas próximas das reais, em seu ambiente de nuvem privado, que é totalmente controlável, razoavelmente barato e onde seus experimentos podem ser testados, avaliados e reproduzidos ao seu dispor. Além de auxiliar o desenvolvimento de pesquisas, a ferramenta também reforçará o caráter reprodutório do seu trabalho, auxiliando futuros pesquisadores a replicar ambientes e testes pré estabelecidos.

\section{Trabalhos Correlatos}

Com o processo de revisão bibliográfica de trabalhos que também abordam o âmbito das nuvens IaaS, não foi encontrado nenhum que realizasse o monitoramento com o objetivo de reprodução de atividades. Dessa forma, é possível dividir os trabalhos correlatos naqueles que se propunham a adquirir as atividades correntes em um sistema IaaS e aqueles se propunham a aplicar cargas sobre tais ambientes.

Dentre os que buscavam monitorar, pode-se listar [Hillbrecht and Bona 2012] e [Xu and Yang 2011], que propõem processos de monitoramento e gestão de máquinas virtuais em tempo real de um sistema de nuvem IaaS de forma remota, fazendo uso do protocolo de SNMP e das ferramentas de coleta de informações e de controle dos hypervisors através da biblioteca libvirt. Outro exemplo é o mecanismo [Corradi et al. 2012], que efetua o monitoramento mútuo de máquinas nós a fim de melhorar a distribuição de criação e migração de máquinas virtuais do escalonador em um ambiente de nuvem IaaS.

Por outro lado, em [Righi et al. 2019], é proposto um sistema de gerenciamento proativo do número CPUs reservadas por um usuário do sistema de nuvem IaaS público por meio da pré alocação e desalocação destes núcleos considerando o consumo deste usuário, desempenho esperado e custo financeiro. Nele, a ferramenta proposta é submetido à cargas flexíveis geradas a partir do processo de integrações numéricas no modelo Newton-Cotes, de forma a simular diferentes cargas de trabalho de $C P U$ sobre o sistema.

\section{Proposta}

A fim de possibilitar a reprodução desejável do consumo de recursos e da comunicação em um ambiente de nuvem, as seguintes métricas serão coletadas: consumo de $C P U$, consumo de memória $R A M$ e tráfego de rede ponto-a-ponto a nível de pacote, definindo tamanho, origem e destino de cada pacote. Estas métricas serão caracterizadas por máquina virtual e por máquina host para que posteriormente possam ser aplicadas sobre um ambiente de nuvem de acordo com as necessidades do pesquisador.

Dentre as opções disponíveis para Hypervisor, exite a bare-metal, onde o hypervisor tem acesso direto ao hardware, e a hosted no qual o gerenciamento suas máquinas virtuais é feito sobre um sistema operacional hospedeiro.

Dada a falta de acesso administrativo a um ambiente de nuvem público, a nuvem privada para a confecção do trabalho terá como gerenciador de nuvem a ferramenta Open Nebula [OpenNebula 2021] e a combinação hypervisor tipo hosted e virtualizador KVM/QEMU[Kivity et al. 2007, Bellard 2005][Bellard 2005]. Quanto ao hardware, as 
máquinas são compostas cada uma por um processador Intel(R) Core $(T M) 2$ Duo $C P U$ E8400@3.00GHz e 4 GB de memória RAM.

O processo de desenvolvimento da ferramenta proposta neste trabalho será dividido em 3 partes: monitoramento de nós, tratamento de dados e aplicação de carga sintética descritas a seguir.

\subsection{Monitoramento de Nós}

O monitoramento de nós será a etapa dedicada à coleta de dados do ambiente de nuvem.

Devido a escolha de um hypervisor tipo hosted, a coleta das métricas será feita através de aplicativos de sonda hospedados no sistema operacional host dos nós host, que efetuará a coleta do consumo de $C P U$ e de memória $R A M$ da máquina host diretamente e indiretamente das máquinas virtuais, pela perspectiva de processos. Além disso, também conta-se com monitores para os pacotes que trafegam pelas placas de rede real e virtuais. Durante todo o processo deverá ser utilizada uma marcação de tempo para indicar o momento em que cada amostra foi coletada. Ao final do processo, os dados do monitoramento deverão ser armazenados em cada uma das máquinas nó, sendo posteriormente coletado para o seu tratamento.

\subsection{Tratamento de Dados}

Nesta etapa será aplicado tratamento aos dados de monitoramento, agrupando-os e caracterizando-os e definindo seus traços por máquina virtual e por máquina host. Desta forma, durante a etapa de reprodução diferentes combinações de máquina virtual ou máquina nó poderão ser aplicadas ao ambiente simulado.

Para tal, serão definidos os valores de consumo de $C P U$, memória $R A M$ e pacotes de rede em intervalos de tempo regulares. Assim, esses traços deverão guiar as aplicações de reprodução de carga no próximo estágio, buscando uma simulação que melhor se aproxime do ambiente monitorado.

\subsection{Aplicação de Carga Sintética}

Nesta etapa será efetuada a aplicação dos traços resultantes da etapa anterior, de modo a guiar as aplicações que proverão carga de trabalho sobre o sistema simulado. Serão definidas máquinas virtuais especializadas para a reprodução da aplicação das cargas. Tais máquinas terão em si aplicações que possam ler os traços e reproduzir a carga de trabalho indicada por intervalo de tempo.

Para efetuar a aplicação de cargas sobre a $C P U$, será definida uma aplicação que efetue operações de integração pelo método de Newton-Cotes [Righi et al. 2019], definindo a função a ser integrada, os parâmetros da função e a área de integração com o decorrer do tempo de simulação, de acordo com o descrito no traço relacionado à $C P U$. Para a reprodução de memória, será definida uma aplicação para alocação e desalocação de memória de acordo com o traço de memória. Finalmente, para a reprodução do tráfego de rede, será utilizada uma aplicação na arquitetura Ping-Pong, de forma a simular a troca de pacotes dos componentes da nuvem entre si e com o mundo.

\section{Avaliação dos Mecanismos}

Para efetuar a avaliação dos mecanismos de coleta de dados e do processo de reprodução, se utilizará das aplicações do projeto NAS Parallel Benchmarks [Saphir et al. 1997] em 
suas versões $M P I$, de forma que os resultados obtidos pelos experimentos estejam de acordo com as medidas esperadas.

\section{Resultados Esperados}

Com a aplicação do processo de monitoramento e de tratamento de dados, espera-se adquirir uma variedade de contextos para as máquinas virtuais, contextos esses que podem ser relacionados com seus pares para reprodução de um ambiente monitorado, ou mesclado com o de outros experimentos, a fim de criar novos e variados cenários para o estágio de reprodução.

Algumas ressalvas devem ser feitas: os traços gerados para o consumo de $C P U$ e memória conterão medidas que descrevem um intervalo de tempo, sendo assim, possíveis usos anômalos desses recursos no ambiente monitorado podem se perder ou ser suavizados durante a modelagem; quanto a rede, como processo se atém apenas ao monitoramento e reprodução nas máquinas reais e virtuais, é possível que demais fatores como roteador entre máquinas, escalonadores de pacotes e a latência da rede possam diferir entre no ambiente monitorado e no simulado, provocando alterações no resultado final. As resoluções ou suavizações tais problemas ainda estão em aberto. Além disso, como o processo de monitoramento em si exige o consumo de recursos das máquinas nó, é esperado que os mecanismos de monitoramento incorram certa interferência e devem ser considerados na aplicação e análise dos resultados dos experimentos.

\section{Referências}

Bellard, F. (2005). Qemu, a fast and portable dynamic translator. In Proceedings of the Annual Conference on USENIX Annual Technical Conference, page 41.

Corradi, A., Foschini, L., Povedano-Molina, J., and Lopez-Soler, J. M. (2012). DDSenabled Cloud management support for fast task offloading. In Symp. on Computers and Communications, pages 67-74.

Hillbrecht, R. and Bona, L. (2012). A snmp-based virtual machines management interface. In Int. Conf. on Utility and Cloud Computing, pages 279-286, Los Alamitos, CA, USA. IEEE Computer Society.

Kivity, A., Kamay, Y., Laor, D., Lublin, U., and Liguori, A. (2007). Kvm: the linux virtual machine monitor. In In Proceedings of the 2007 Ottawa Linux Symposium (OLS'-07.

Mell, P. and Grance, T. (2011). The NIST Definition of Cloud Computing. National Institute of Standards and Technology Special Publication 800-145.

OpenNebula (2021). Opennebula. https://opennebula.io/. Acessado em: 25/02/2021.

Righi, R. D. R., Rodrigues, V., Nardin, I., Costa, C., Alves, M. A. Z., and Pillon, M. A. (2019). Towards providing middleware-level proactive resource reorganisation for elastic HPC applications in the cloud. Int. Journal of Grid and Utility Computing, 10:76-92.

Saphir, W., der Wijngaart, R. V., Woo, A., and Yarrow, M. (1997). New Implementations and Results for the NAS Parallel Benchmarks 2. In PPSC.

Xu, L. and Yang, J. (2011). A management platform for Eucalyptus-based IaaS. Int. Conf. on Cloud Computing and Intelligence Systems, pages 193-197. 